\title{
Chatbot as a New Business Communication Tool: The Case of Naver TalkTalk
}

\author{
Miri Heo, Kyoung Jun Lee
}

School of Management, Kyung Hee University, Seoul, Korea

Objectives: Chatbot is a program that talks in voice or text. It is equipped with artificial intelligence (AI) technology and is developed in a way to combine with messenger, offering companies new opportunities for business communication. Companies expect cost savings and sales increases by providing chatbot-based services to their customers, such as providing responses to inquiries and fulfilling orders without time constraints. However, the current absence of conversationally perfect AI technology creates a climate in which the public holds unreasonable expectations, as businesses fail to understand the current status of AI and the marketing of chatbot technology companies is hyped.

Methods: This is a single-case study. While looking for a successfully deployed chatbot, we found Naver TalkTalk, a card-based chatbot application. We interviewed Naver's managerial and technical staff four times and acquired internal data as well as external data. Results: We illustrate Naver TalkTalk, a successful card-based business chatbot, and examine how Naver TalkTalk has developed and evolved for effective business communication. The first chatbot started without automation and then failed in the process of evolving to automation. This chatbot was changed to cardbot and was re-automated based on the card-based interface and robotic process automation (RPA).

Conclusions: The cardbot-based chatbot is quick and easy to handle, which is why it has been popular among users. It is now becoming a new business communication channel to attract new customers. In addition, the proper automation of business communication is increasing efficiency and reducing costs of businesses.

Key Words: Chatbots, Business Chatbots, Conversation, Robotic Process Automation (RPA), Artificial Intelligence (AI)

\section{Introduction}

With the advent of smartphones, the core of the Internet market has shifted from existing web browsers to mobile platforms and

Received: Oct 20, 2017 Revised: Nov 13, 2017 Accepted: Nov 20, 2017

Corresponding author: Kyoung Jun Lee

School of Management, Kyung Hee University, 26 Kyungheedae-ro,

Dongdaemun-gu, Seoul 02447, Korea

Tel: +82-2-961-0490, E-mail: klee@khu.ac.kr

This is an Open Access article distributed under the terms of the Creative Commons Attribution Non-Commercial License (http://creativecommons.org/licenses/by$\mathrm{nc} / 4.0 /$ ) which permits unrestricted non-commercial use, distribution, and reproduction in any medium, provided the original work is properly cited. Copyright $\odot 2018$ Korean Association for Business Communication. a variety of application ecosystems. However, as major mobile platform companies such as KakaoTalk in Korea, Tencent in China, Line in Japan, and Whatsapp in the United States established messaging applications as mid- and long-term growth engines, in the future, the chatbot-based messaging app is expected to become a strong alternative to the current web browser's role. Chatbot allows users to chat in voice or text (a computer program that conducts a conversation via auditory or textual methods) (Mauldin, 1994) in spite of the fact that artificial intelligence technology that enables perfect conversation has yet to be sufficiently developed. Currently, there is a mix of an excessive expectation of the public and companies who do not understand the technology status of chatbots as well as the hyped 
marketing of chatbot-related companies. We have not yet found a fully automated chatbot that was practically effective (Heo \& Lee, 2017) except for the so-called cardbot, which was first defined by Heo and Lee (2017) as a chatbot with multiple-choice cards as its main user interface form.

While looking for a successfully deployed chatbot, we found Naver TalkTalk, a card-based chatbot application. We investigated this case in a single-case study. As a research method, we interviewed Naver's managerial and technical staff four times and acquired internal as well as external data. In this study, we investigate how Naver TalkTalk has transformed and evolved and draw implications for the successful development and management of chatbots.

\section{Case Description}

Naver Inc. has an Internet portal 'NAVER.com,' which is topranked in Korea, and a global mobile messenger platform 'LINE', which is ranked first in Japan and second in Korea. Based on a variety of Internet services, Naver offers a wide range of services including banner advertising, keyword search advertising, video advertising, online shopping, Naver Pay, cloud service, and Naver music. Naver has a shopping-related platform called 'Store Farm. Naver started to develop TalkTalk to support the online shopping of offline stores such as 'Hongdae Fashion' and 'Apgujeong Style, which can be regarded as an $\mathrm{O} 2 \mathrm{O}$ (Online-to-Offline) service.

\section{Chatting by and between Humans}

In January 2015, in order to display an offline store's products online and realize the $\mathrm{O} 2 \mathrm{O}$ service, Naver created a platform for simply uploading pictures of goods that can be seen at the store. It succeeded in attracting the attention and the visits of potential consumers. However, the people visiting the offline stores did not remain in Naver's business customer's online shops. Contrary to what they envisioned, the online relationship between the consumers and the stores was established through KakaoTalk, the main competitor of Naver Line. As a result, online inquiries were also being processed via KakaoTalk, and valuable consumer information was being transferred to Kakao. Naver's TalkTalk emerged from this desperate situation.

When a customer found a product she or he wanted to see, the customer could easily ask for the price or size through con- versation with the offline seller, as depicted in Figure 1 (Kim, 2017). Customers were able to communicate through direct conversation and purchase the product at the negotiated price. To support this business communication model between offline sellers and online consumers, the TalkTalk was developed.

Unlike a typical price-fixed online shopping mall, customers can get a discount through conversation without having to set a price for the product and sometimes offer it at a customized price. At the time of its first implementation, it was more successful at a local shop outside of Seoul, the capital of Korea, with sales of over 100 million Korean Won per month in four months.

Naver TalkTalk used a different approach from web-based Q\&A or FAQ services on existing online shopping sites. It was a good idea to incorporate a chat service that allows users to solve questions while shopping. As a smartphone user's most popular app is a messaging app, it has become a marketing tool for companies to manage their customer relationships. However, from the buyer's side, they did not want to feel burdened by being "friends" with the company's official account and receiving marketing messages from them. Naver's TalkTalk has the advantage of being able to inquire directly to the store owner without the hassle of adding a messenger buddy or a friend on another messaging app, which means that the consumers did not need to have the burden of receiving unwanted information.

However, from the business side, a new problem emerged. During an interview about the secret of success, sellers expressed the opinions that "chatting is as much of a burden as selling." In addition to serving staff working at the offline store, they had to hire online chatting staff and made them chat until dawn. Naver started to wonder how to save the seller time. They considered but did not use the Q\&A function by making bullets for frequently used phrases or simple FAQ parts. If so, the questions could be reduced to some extent, but they did not think it was very effective.

\section{Trying Chatting Automation}

In June 2015, Naver opened a shopping bot to maintain merchants' sales volume while reducing chat consultation. Along with Naver's natural language processing laboratory, they have developed a chatbot that recognizes and responds to natural language. At the time of the first chatbot, simple conversations based on natural language processing responded well, but communication was not good when asked with slightly more diffi-

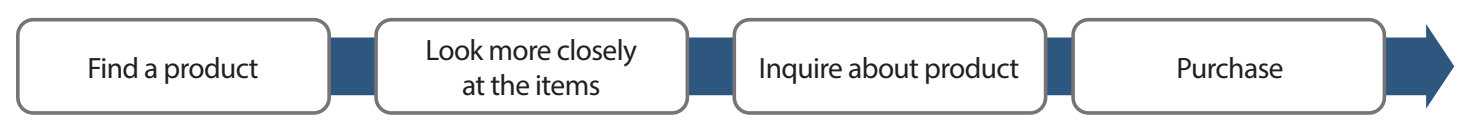

Figure 1. Flow of Shopping Users (Kim, 2017). 
cult questions. In the daytime, the seller responded directly and used the shopping bots at night or during the holidays.

The successful response rate was only $7 \%$, and the conversion rate of purchasing with it was just $0.1 \%$. The chatbot is technically new and exciting, which is of course important, but the shopping bots have not been able to successfully sell products even though they literally have a concrete business objective, which is to sell products for the sellers.

Furthermore, most of the consumer respondents answered "I do not know what to ask." The chatbot says "ask any questions!" but users do not know what to ask. They say "It is good to use messenger when talking to a friend, but it is rather cumbersome and inconvenient when talking to chatbot." In fact, as stock and
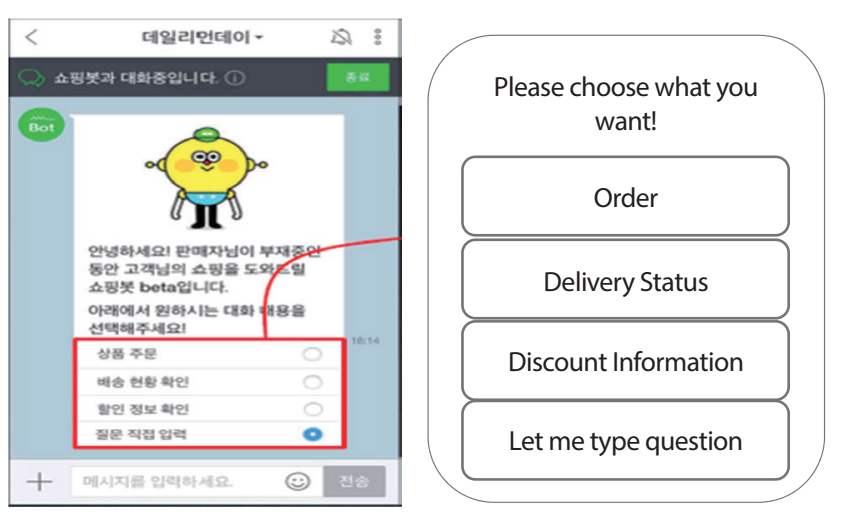

Figure 2. A Typical Cardbot Interface. size inquiries were listed in the product description, there was no reason for users to type an inquiry.

\section{Evolving to Cardbot}

In September 2015, Naver launched a cardbot that allows shopbots to specify what they can do reflecting buyer's needs. The selection scenarios in the cardbots were divided into categories based on the most frequently asked questions, as depicted in Figure 2.

In the first month since the implementation of the cardbots, the company achieved success with a $29 \%$ compliance rate and $12.4 \%$ purchase conversion. Naver realized through experience that chatbots do not work if they demand only natural language, and they evolved to a service that can provide users with scenarios and let users choose among them. By doing so, the response rate rose. The shopping bots have also been actively operated 24/7. Naver finally realized that letting users type natural words in text did not work well. Instead, they found that letting users choose a scenario is practically successful when a customer selects simple automation functions such as ordering and delivery date inquiry.

Since then, Naver continued to develop the chatbot platform with Chatbot API and provided it to several companies internally by the closed beta method, and it is now open API. It is a message API similar to that of Facebook and KakaoTalk API.

Another successful example of a cardbot is a delivery-bot. In
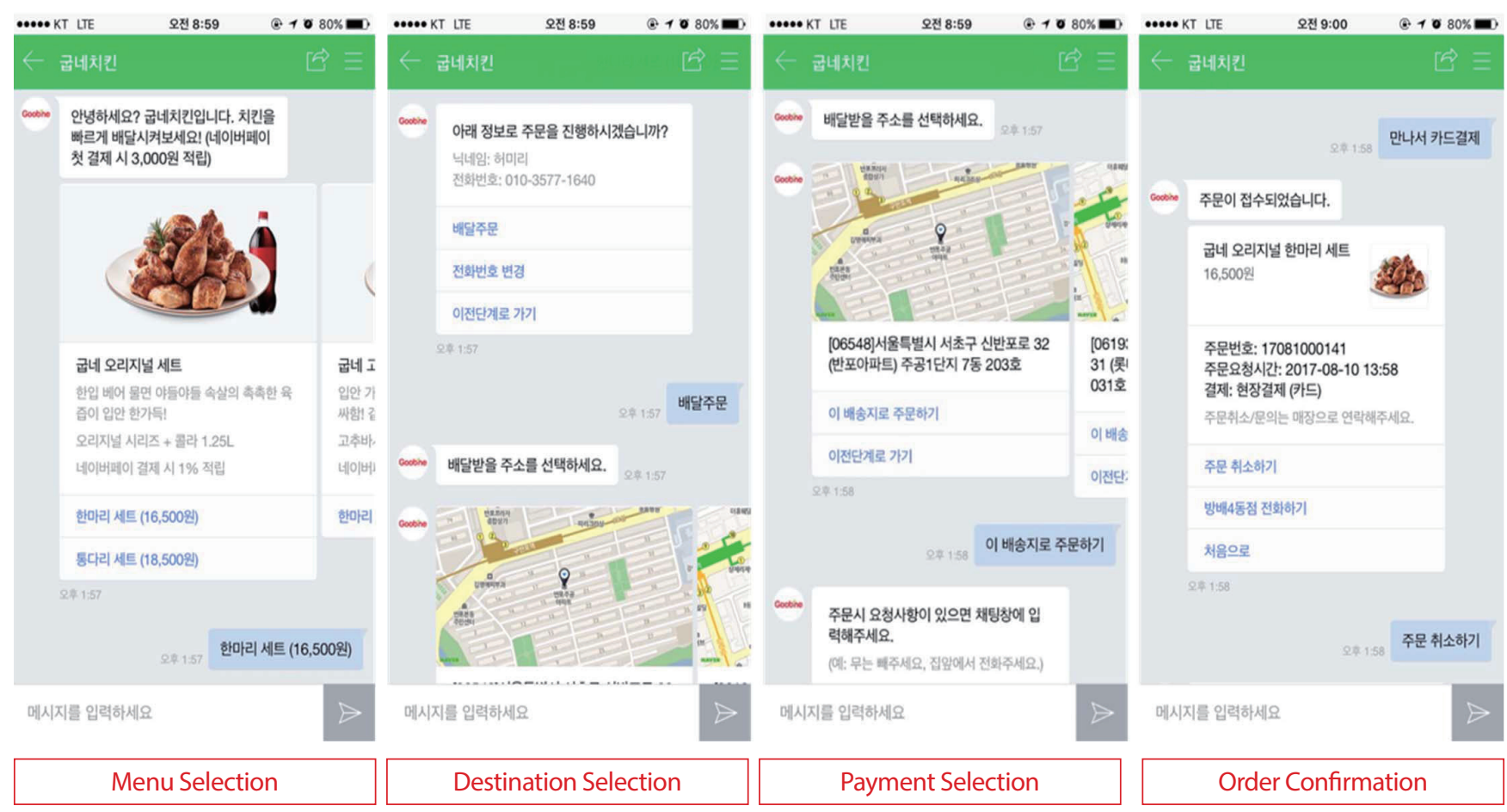

Figure 3. Four Steps for Delivery-bot Ordering. 
the summer of 2016, Domino Pizza wanted to try a new marketing method through chatbot and wanted to reduce the burden on the customer center's phone orders. Naver surveyed and analyzed various order-related chatbots, and they launched the delivery-bot in February 2017, which considered how to sell a higher quantity of pizza. Naver minimized the order steps through customized customer information data so that orders could be made with only four touches including menu selection, destination selection, payment method selection, and order confirmation. Customers could place orders by clicking on the simple order button that comes up when you search for the name of the business providing the order in the Naver search window or by checking the location of the store based on the map provided in the nearby store search results, as depicted in Figure 3.

Buyers liked the cardbot service because it is 'simple and quick.' A buyer said 'I was not burdened because the robot responded not to people, but it was good to order without a login process.' Naver made it easy for customers to choose what they needed right away, and they could now place an order with just a touch without worrying.

Domino announced that $66 \%$ of the total order volume was from new customers. Domino thinks it would be good to use the bot as a new menu or event menu. Since launching TalkTalk, which is cardbot based, it has increased to 50,000 operators in January 2016, increased to 110,000 in July and 160,000 in December 2016, and the chatbot service has expanded its scope not only to shopping companies but also to various small offline businesses. In addition, there are more and more business cases being developed through the chatbot API such as credit card recommendation bots, cooking recommendation bots, hospital bots, and laundry bots, and the fun and interest-oriented chatbots.

\section{Discussion}

Some people did not make choices even though they had a menu to choose from (54.5\%). Some people just typed in natural language, and in that case the cardbot was required to analyze the user's natural language through a machine learning technique and connect it to one of the prepared menus, as depicted in Figure 4.

Although the shopping bot service was successful, only $30 \%$

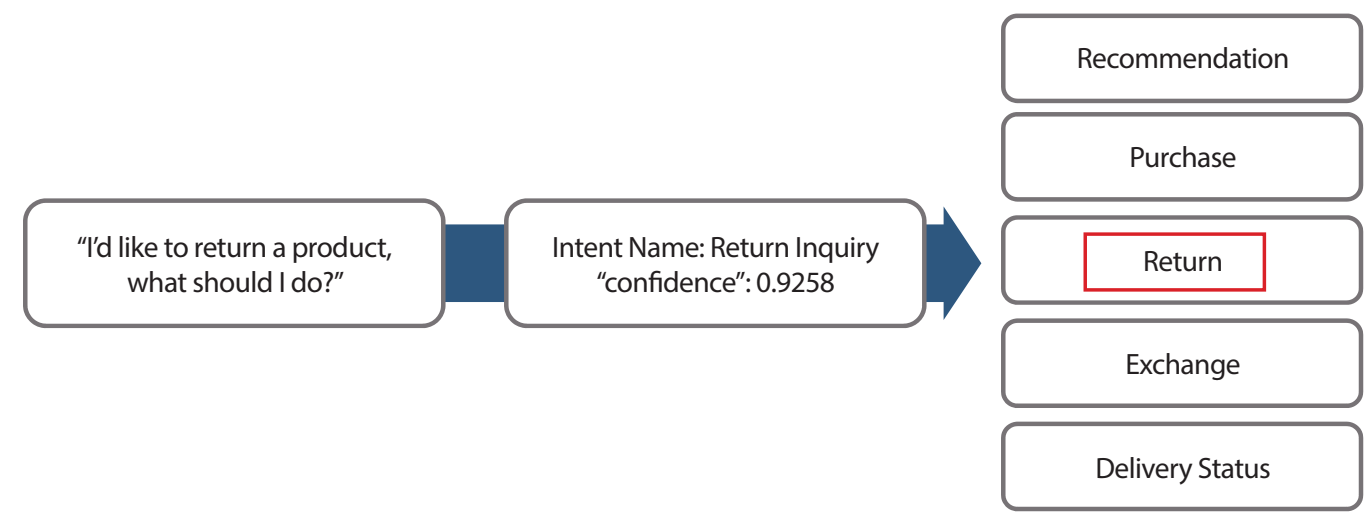

Figure 4. Matching a natural language question with prepared menu in robotic process automation.
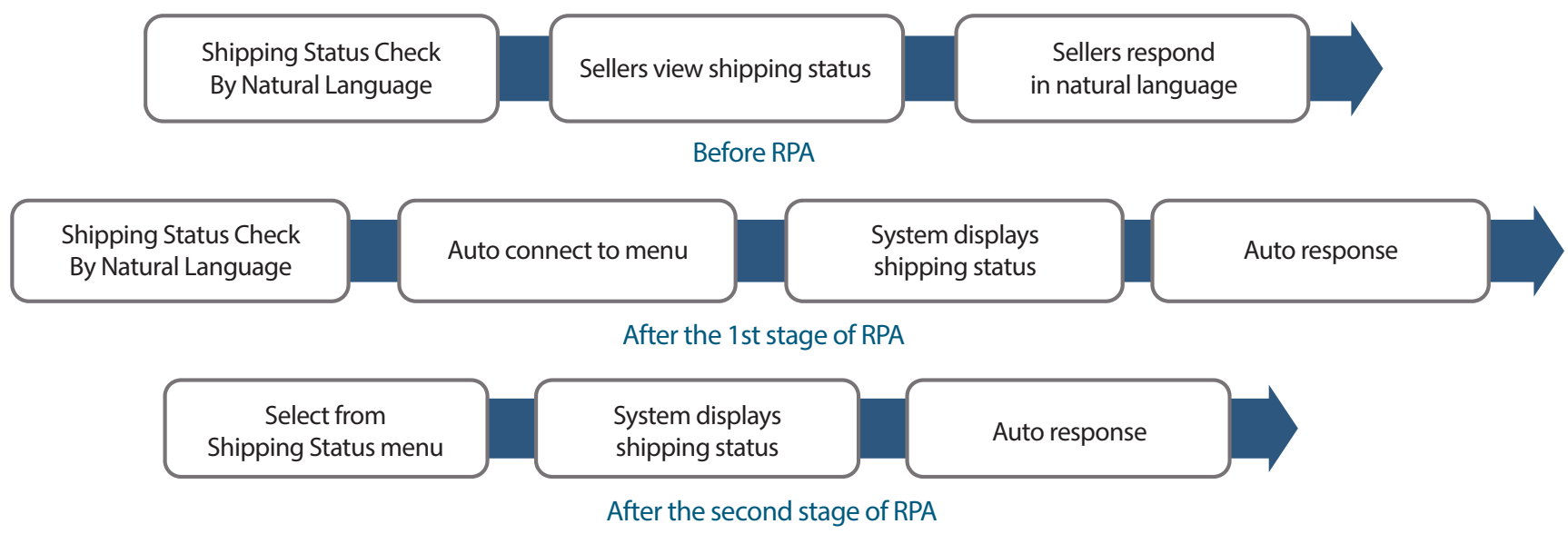

Figure 5. The Steps of robotic process automation (RPA). 
have been automated. The sales department began to feel the need for robotic process automation (RPA). RPA is a softwarebased approach to automatically perform the kinds of administrative tasks that otherwise require stop-gap human handling (Lacity \& Willcocks, 2015). Naver aims to increase the automation rate by $70 \%$ in the future by extracting thousands of actual user inquiries about shopping intent, as depicted in Figure 5. Currently, there are five scenarios available, but they plan to increase it to eight and make it easier. Such automation is not only a matter of cost reduction but also has a considerable impact on customer satisfaction. For example, if a robot responds to hotel guests at a hotel, the people can demand more services and increase their level of satisfaction.

In the case of shopping bots, most of the repetitive business processes such as delivery status, size inquiry, and exchange/refund etc. are most likely to be automated by RPA. In the future, it will be necessary to develop a step-by-step plan that enables people and AI to implement automation strategies in collaboration.

So far, there has been a lack of dialogue data that can be applied to machine learning. But the shopping and delivery bots are simple enough to collect data where chatbots could not respond automatically, therefore adding the pattern to the learning data set. The Naver TalkTalk chatbot platform is also developing chatbots that can be developed by small business owners so that they can develop and participate in data collection early on. The bots were used early for e-commerce business. Now the number of offline service providers using chatbots is increasing, which is expected to greatly contribute to small businesses and street merchants.

\section{Conclusion}

In this study, we found that customers who use chatbots feel great value for their quickness and confirmed the possibility of using new chatbots as a means of securing new customers and launching new services. However, some vendors who do not have clear performance targets for chatbots are found to experi- ence difficulties, and it is necessary to develop chatbots that meet clear performance targets and the characteristics of the vendor.

Chatbot is quick and easy to handle, which makes increases customer satisfaction. It is now becoming a new business communication channel to attract new customers. In addition, the proper automation of business communication is increasing efficiency and reducing the costs of businesses.

\section{Acknowledgement}

We would like to express our sincere appreciation to Mr. Taeeun Kang and Mr. Hyunsoo Jung of Naver Inc. for kindly allowing and participating in our interview and providing us with the data for this case report.

This work was supported by the Ministry of Education of the Republic of Korea and the National Research Foundation of Korea (NRF-2017S1A5B8059804).

\section{References}

Mauldin, M. L. (1994). ChatterBots, TinyMuds, and the Turing Test: Entering the Loebner Prize Competition. Proceedings of the Eleventh National Conference on Artificial Intelligence (pp. 16-21). Palo Alto, CA: AAAI Press.

Heo, M., \& Lee, K. (2017). Case analysis of chatbots as business communication methods. Proceedings of the 2017 Summer Korea-Japan Joint Conference on Business Communication (pp. 51). Gwangju, Korea.

Kim, J. (2017, August). Naver TalkTalk. Paper presented at the joint conference of the 19th International Conference on Electronic Commerce and the 2017 Spring Conference of Korea Intelligent Information Systems Society, Pangyo, Korea.

Lacity, M. C., \& Willcocks, L. (2015). What knowledge workers stand to gain from automation. Harvard Business Review. Retrieved from https://hbr.org/2015/06/what-knowledge-workers-stand-to-gainfrom-automation 